\title{
Electrical energy storage and dissipation in materials
}

\author{
Xiaoyu Zheng \\ Department of Mathematical Sciences, Kent State University, Kent, Ohio, 44224 \\ Peter Palffy-Muhoray \\ Liquid Crystal Institute, Kent State University, Kent, Ohio, 44224
}

April 3, 2015

\begin{abstract}
Using a simple classical approach, we consider where and how electrical energy is stored in lossy dispersive materials. We argue that the material contribution to the electrical energy density is simply the sum of the kinetic and potential energies of the charges present under the influence of the applied electric field. It follows that the stored energy density must be positive. We provide simple expressions for the stored and dissipated energy densities; in the lossless case, our expressions reduce to the standard results of Brillouin and Landau.
\end{abstract}

Keywords Electrical energy density; lossy dispersive materials

\section{Introduction}

Due to the increased use of sophisticated electronic and optical devices, the storage of electrical energy is an increasingly important practical problem $[1,2]$. Batteries store electrochemical energy. Electrical energy is typically stored in capacitors containing dielectric materials, and the design of dielectrics for high density energy storage is a very active area of materials research today $[3,4,5]$. Electrical energy needs to be stored (semi) permanently, in devices using DC, as well as temporarily, in devices using AC and electromagnetic radiation. Energy storage is also of interest in studies of nanomaterials for negative index and cloaking applications $[6,7,8,9]$. Frequencies of interest thus range from DC to optical frequencies.

Exactly where and how electrical energy is stored in matter are simple but interesting questions with some surprising answers.

It is commonly believed, and, as discussed by Feynman [10], it is almost certainly true, that, in vacuum, at each point in space, the electric field $\mathbf{E}$ has energy, and the energy density is given by

$$
W_{0}=\frac{1}{2} \varepsilon_{0} E^{2},
$$

where $\varepsilon_{0}$ is the permittivity of free space. The power $\mathcal{P}_{f}$ associated with change of this energy density in volume $V$ is

$$
\frac{\mathcal{P}_{f}}{V}=\frac{\partial W_{0}}{\partial t}=\varepsilon_{0} \mathbf{E} \cdot \dot{\mathbf{E}} .
$$

Now if ordinary matter is placed in this electric field, say between the plates of a capacitor at some given voltage, the energy density changes, even though the electric field remains the same. Why should this be so, given that ordinary matter is mostly empty space, essentially vacuum, with the same field as before?

Many, perhaps most, textbooks on electricity and magnetism and optics tell us that the energy density of the electric field in matter is (e.g. [11]),

$$
W_{s}=\frac{1}{2} \mathbf{D} \cdot \mathbf{E}=\frac{1}{2} \varepsilon E^{2},
$$

where $\varepsilon=\varepsilon_{0} \varepsilon_{r}$ is the dielectric permittivity of the material, and $\mathbf{D}=\varepsilon \mathbf{E}$ is the electric displacement. This simple and beautiful result holds in case of static fields or lossless and non-dispersive media, where $\varepsilon$ is real 
and independent of frequency, but it is not true in general. An illustrative example of where it does not hold is in the case of metals, where, in the Drude description [12, 13, 14], ignoring dissipation, $\varepsilon$ is given by

$$
\varepsilon=\varepsilon_{0}\left(1-\frac{\omega_{p}^{2}}{\omega^{2}}\right)
$$

where $\omega$ is the angular frequency of the electric field, and $\omega_{p}$ is the plasma frequency. Since for frequencies below the plasma frequency $\varepsilon$ is negative, the simple expression of Eq. (3) gives a negative energy density. This is clearly unphysical, since if the energy with a field were to be lower than that without, then charges would spontaneously organize to create such a field - which, as we know from experience, does not occur.

The question then becomes what is the correct expression for the energy density of the electric field in a medium where the dielectric constant is a function of frequency - that is, in ordinary matter. The expression for the electric energy density in dispersive materials is not readily found in textbooks, and, as current literature indicates, there is no consensus on what is the correct expression.

For non-dissipative materials, Landau gives the average stored energy density as [15],

$$
\bar{W}_{s}=\frac{1}{2} \frac{\partial(\omega \varepsilon)}{\partial \omega}\left\langle E^{2}\right\rangle
$$

based on prior work by L. Brillouin $[16,17] .{ }^{1}$ This important result is not widely known, and an elementary derivation is not easily readily accessible. For dissipative dispersive materials, conflicting results exist in the literature.

\subsection{Energy Density Results in the Literature}

Most derivations of the expression for stored electric energy density are based on Poynting's theorem and the conservation energy [18, 19, 20, 8, 21]. From Poynting's theorem, it follows that the rate of work done by the electric field $\mathbf{E}$ on the system - consisting of the material and the space containing it - per unit volume, is given by $[22,23]$,

$$
\frac{\mathcal{P}_{i T}}{V}=\mathbf{E} \cdot \dot{\mathbf{D}}
$$

Here $\mathcal{P}_{i T}$ is the total input power delivered by the electric field into volume $V$. It is straightforward to compute the input power from Eq. (6). From the conservation of energy, it follows that the input power must equal the sum of the power dissipated by the system (in heat, radiation, etc.) and the rate of change of electrical energy stored in the system. The difficulty lies in the partitioning of the input power into these two distinct components - the dissipation rate and the rate of change of stored energy.

There appears to be two approaches to identifying these components. One approach, which we call microscopic, considers energy storage and dissipation in the material in terms of processes at the microscopic level $[18,19,20,8,21]$, or equivalently, in terms of equivalent circuits $[24,7,25]$. The alternate approach, which we call macroscopic [22, 26, 9, 27], considers only macroscopic fields and bulk response functions. The separation of dissipated power from the rate of change of stored energy is based on the characteristic forms of expressions associated with these.

The results of the microscopic approaches cited all agree with one another, and give one expression for the stored energy. The average stored energy density from microscopic approaches can be written concisely as $[25]$

$$
\bar{W}_{s}^{\text {micro }}=\frac{1}{2} \varepsilon_{0}\left(1+\left|\frac{\partial \omega \chi(\omega)}{\partial \omega}\right|\right)\left\langle E_{0}^{2} \cos ^{2}(\omega t)\right\rangle .
$$

Here $\chi(\omega)=\varepsilon(\omega) / \varepsilon_{0}-1$, and the notation $|\cdot|$ indicates the magnitude of a complex argument. The stored energy density is always positive, as explicitly required by Landau [15] from general considerations, and by the microscopic theories $[17,18,19,20,8,21]$.

The results of the macroscopic approaches also agree with one another, but give a different expression for the stored energy. The average stored energy density from macroscopic approaches can be written concisely as $[22,9,27]$

$$
\bar{W}_{s}^{\text {macro }}=\frac{1}{2} \varepsilon_{0} \operatorname{Re}\left(1+\frac{\partial \omega \chi(w)}{\partial \omega}\right)\left\langle E_{0}^{2} \cos ^{2}(\omega t)\right\rangle
$$

\footnotetext{
${ }^{1}$ Note that using the Drude expression for $\varepsilon$ for metals in Eq. (4) here results in a positive energy density.
} 
which clearly differs from Eq. (7). According the Eq. (8), the macroscopic approach allows the stored energy to be negative, as claimed explicitly in Ref. [9]. This is at variance with microscopic theories, as well as the general considerations of Landau. In addition, we reiterate that if the energy density with a field were to be lower than that without, as negative stored energy density implies, then charges would spontaneously organize to create such a field - which does not occur. We therefore regard Eq. (8) to be incorrect.

In the following section, we derive the expression for the electric energy density of dispersive and lossy materials through an exceedingly simple and straightforward microscopic approach, simpler than what currently exists in the literature. Neither Maxwell's equations nor Poynting's theorem is involved in our derivation; we argue that contributions of the material to the stored energy density can be simply understood in terms of changes of the classical kinetic and potential energies of the electrons in the material caused by the applied electric field. In addition, we point out incorrect assumptions in the literature, and conclude that the material contribution to the electric energy density must be positive definite.

\section{Energy storage and dissipation in dispersive media}

We begin with the result [10] that, in vacuum, at each point in space, the electric field $\mathbf{E}$ has energy, and the energy density is given by

$$
W_{0}=\frac{1}{2} \varepsilon_{0} \mathbf{E}^{2} .
$$

Here $\mathbf{E}$ may be visualized as the electric field between the plates of a capacitor, in vacuum, connected to a voltage source which maintains the voltage. Now if some homogenous material were to fill the space between the capacitor plates, then the macroscopic electric field will remain unchanged, since the voltage is unchanged. Since ordinary condensed matter is mostly empty space (volume of nucleus/volume of atom $\sim 10^{-14}$ ), the energy density due to the field $\mathbf{E}$ should remain essentially unchanged. If there is a change in the energy density, it can only come from changes in the energies of the particles in the material. Since $\mathbf{E}$ exerts a force on charges, the change in energy stored by the electric field can only come through changes in the kinetic and potential energies of charges in the material. We therefore consider the motion of charges under the influence of the electric field.

Classical atoms can give valuable insights in many situations [28]. In the Lorentz model, the electrons are considered to be decoupled harmonic oscillators [14]. Since the electric field exerts no net force on a neutral atom, the center of mass remains at rest, and since the mass of the nucleus $m_{n}>m_{e} \times 10^{3}$, where $m_{e}$ is the mass of the electron, we assume that the field induced motion of the nuclei can be neglected.

The equation of motion of the electrons is then

$$
m_{e} \ddot{x}+\beta \dot{x}+k x=q E(t),
$$

where $q$ is the charge, $\beta$ is an effective friction coefficient describing dissipation, and $k \sim \omega_{p}^{2} m_{e} / 3$ is an effective spring constant. We assume the local electric field acting on the electrons to be essentially the same as the average Maxwell field $\mathbf{E}(t)$. We now multiply Eq. (10) through by the velocity $\dot{x}$ and obtain

$$
m_{e} \ddot{x} \dot{x}+\beta \dot{x}^{2}+k x \dot{x}=E(t) q \dot{x} .
$$

The first term on the left side of Eq. (11) is the rate of change of the kinetic energy of the electron, the second is the dissipated power and the third is the rate of change of potential energy. The right side is the input power delivered by the electric field.

Multiplying Eq. (11) by the number density $\rho$ of electrons, we get

$$
\rho m_{e} \ddot{x} \dot{x}+\rho \beta \dot{x}^{2}+\rho k x \dot{x}=E(t) \rho q \dot{x},
$$

and since the electric polarization $P=\rho q x$, this is

$$
\frac{\partial}{\partial t}\left(\frac{K \mathcal{E}}{V}\right)+\frac{\mathcal{P}_{d}}{V}+\frac{\partial}{\partial t}\left(\frac{P \mathcal{E}}{V}\right)=\mathbf{E} \cdot \dot{\mathbf{P}}=\frac{\mathcal{P}_{i}}{V}
$$

where $V$ indicates volume. So $\mathbf{E} \cdot \dot{\mathbf{P}}$, the instantaneous power transferred to the material by the field, per volume, is equal to the rate of change of stored energy density, plus the dissipated power density. 
Next, we describe the motion using complex notation, recalling that physical quantities are represented by the real parts of complex quantities. If $E(t)=E_{0} e^{-i \omega t}$, then the solution of Eq. (10) is

$$
x=\frac{q E / m_{e}}{\omega_{0}^{2}-\omega^{2}-i \gamma \omega},
$$

where $\omega_{0}=\sqrt{k / m_{e}}$ is the resonance frequency and $\gamma=\beta / m_{e}$. We multiply Eq. (14) through by the charge density $\rho q$, and, if all electrons obey the same equation of motion, we obtain

$$
P=\frac{\omega_{p}^{2}}{\omega_{0}^{2}-\omega^{2}-i \gamma \omega} \varepsilon_{0} E=\chi(\omega) \varepsilon_{0} E
$$

where $\omega_{p}^{2}=\rho q^{2} / m_{e} \varepsilon_{0}$ is the plasma frequency and $\chi$ is the susceptibility. We note that $\mathbf{D}=\varepsilon_{0} \varepsilon_{r} \mathbf{E}=\varepsilon_{0} \mathbf{E}+\mathbf{P}$, and solving for $\varepsilon_{r}$, we get

$$
\varepsilon_{r}=1+\frac{\omega_{p}^{2}}{\omega_{0}^{2}-\omega^{2}-i \gamma \omega}=\varepsilon_{r}^{\prime}+i \varepsilon_{r}^{\prime \prime}
$$

We now give explicit expressions for the energy densities and power in terms of electrical quantities; these are as follows.

The potential energy density $P \mathcal{E} / V=\frac{1}{2} \rho k(\operatorname{Re}(x))^{2}$ is

$$
\frac{P \mathcal{E}}{V}=\frac{1}{2} \varepsilon_{0} E_{0}^{2} \frac{\omega_{0}^{2}}{\omega_{p}^{2}}\left[\frac{1}{2}\left(\left(\varepsilon_{r}^{\prime}-1\right)^{2}+\varepsilon_{r}^{\prime \prime 2}\right)+\frac{1}{2}\left(\left(\varepsilon_{r}^{\prime}-1\right)^{2}-\varepsilon_{r}^{\prime \prime 2}\right) \cos 2 \omega t+\left(\varepsilon_{r}^{\prime}-1\right) \varepsilon_{r}^{\prime \prime} \sin 2 \omega t\right],
$$

and the kinetic energy density $K \mathcal{E} / V=\frac{1}{2} \rho m_{e}(\operatorname{Re}(\dot{x}))^{2}$ is

$$
\frac{K \mathcal{E}}{V}=\frac{1}{2} \varepsilon_{0} E_{0}^{2} \frac{\omega^{2}}{\omega_{p}^{2}}\left[\frac{1}{2}\left(\left(\varepsilon_{r}^{\prime}-1\right)^{2}+\varepsilon_{r}^{\prime \prime 2}\right)-\frac{1}{2}\left(\left(\varepsilon_{r}^{\prime}-1\right)^{2}-\varepsilon_{r}^{\prime \prime 2}\right) \cos 2 \omega t-\left(\varepsilon_{r}^{\prime}-1\right) \varepsilon_{r}^{\prime \prime} \sin 2 \omega t\right] .
$$

and the density of energy stored in the field $E, P \mathcal{E}_{E} / V$ is

$$
\frac{P \mathcal{E}_{E}}{V}=\frac{1}{2} \varepsilon_{o} E_{o}^{2}\left(\frac{1}{2}+\frac{1}{2} \cos 2 \omega t\right) .
$$

As shown in Eqs. (17) and (18), the stored kinetic and potential energies, and their sum, oscillate in time. It is more practically useful therefore to consider their time averages. For the total average stored energy density $\bar{W}_{s}$ we obtain

$$
\bar{W}_{s}=\left\langle\frac{P \mathcal{E}_{E}}{V}\right\rangle+\left\langle\frac{P \mathcal{E}}{V}\right\rangle+\left\langle\frac{K \mathcal{E}}{V}\right\rangle=\frac{1}{4} \varepsilon_{0} E_{0}^{2}\left(1+\frac{\omega_{p}^{2}\left(\omega_{0}^{2}+\omega^{2}\right)}{\left(\omega_{0}^{2}-\omega^{2}\right)^{2}+\gamma \omega^{2}}\right),
$$

where the brackets $\langle\cdot\rangle$ indicate time averages, and we have included, in the first term on the right, the energy density due to the electric field in vacuum. Interestingly, the total average stored energy density can be written as

$$
\bar{W}_{s}=\frac{1}{4} \varepsilon_{0} E_{0}^{2}\left(1+\left|\frac{\partial \omega \chi}{\partial \omega}\right|\right),
$$

which, in the lossless case, agrees with Eq. (5) and Refs. [15, 16, 17].

\subsection{Power and Dissipation}

The input power per volume to the material, $\mathcal{P}_{i} / V=\mathbf{E} \cdot \dot{\mathbf{P}}$, is

$$
\frac{\mathcal{P}_{i}}{V}=\mathbf{E} \cdot \dot{\mathbf{P}}=\frac{1}{2} \varepsilon_{0} E_{0}^{2} \omega\left(-\left(\varepsilon_{r}^{\prime}-1\right) \sin 2 \omega t+\varepsilon_{r}^{\prime \prime}+\varepsilon_{r}^{\prime \prime} \cos 2 \omega t\right) .
$$

The total input power $\mathcal{P}_{i T}$, which includes the power to change the energy stored in the field $E(t)$, is 


$$
\frac{\mathcal{P}_{i T}}{V}=\mathbf{E} \cdot\left(\varepsilon_{0} \dot{\mathbf{E}}+\dot{\mathbf{P}}\right)=\mathbf{E} \cdot \dot{\mathbf{D}}=\frac{1}{2} \varepsilon_{0} E_{0}^{2} \omega\left(-\varepsilon_{r}^{\prime} \sin 2 \omega t+\varepsilon_{r}^{\prime \prime}+\varepsilon_{r}^{\prime \prime} \cos 2 \omega t\right) .
$$

Since the quantity $\varepsilon_{r}^{\prime}$ is often associated with stored energy and $\varepsilon_{r}^{\prime \prime}$ with dissipation, it is tempting to assume that the first term on the right side of Eq. (23) gives the rate of change of stored energy, while the second and third give the dissipated power. This, however, is not the case, as we show explicitly below.

The dissipated power per volume, $\mathcal{P}_{d}=\rho \beta(\operatorname{Re}(\dot{x}))^{2}=\gamma \omega_{p}^{2}(\operatorname{Re}(\dot{P}))^{2} / \varepsilon_{0}$, is

$$
\frac{\mathcal{P}_{d}}{V}=\frac{1}{2} \varepsilon_{0} E_{0}^{2} \gamma \frac{\omega^{2}}{\omega_{p}^{2}}\left[\left(\left(\varepsilon_{r}^{\prime}-1\right)^{2}+\varepsilon_{r}^{\prime \prime 2}\right)-\left(\left(\varepsilon_{r}^{\prime}-1\right)^{2}-\varepsilon_{r}^{\prime \prime 2}\right) \cos 2 \omega t-2\left(\varepsilon_{r}^{\prime}-1\right) \varepsilon_{r}^{\prime \prime} \sin 2 \omega t\right] .
$$

Energy conservation given in Eq. (13) can be verified by direct substitution. We point out here that it is evidently not true that

$$
\mathcal{P}_{d} / V=\mathcal{P}_{i T} /\left.V\right|_{\varepsilon_{r}^{\prime}=0}
$$

or that

$$
\partial P \mathcal{E} / V \partial t+\partial K \mathcal{E} / V \partial t+\partial P \mathcal{E}_{E} / V \partial t=\mathcal{P}_{i T} /\left.V\right|_{\varepsilon_{r}^{\prime \prime}=0}
$$

at each instant in time, as claimed in the literature [9].

We also note that the time dependent dissipation cannot be simply written as

$$
\frac{\mathcal{P}_{d}}{V}=\omega \varepsilon_{0} \varepsilon_{r}^{\prime \prime} E_{0}^{2} \cos ^{2} \omega t,
$$

as proposed by Jackson [22]. The results of the macroscopic approaches, resulting in Eq. (8) and allowing negative energy densities ${ }^{2}$ therefore do not hold.

On taking time averages, however, we do find that

$$
\left\langle\mathcal{P}_{d}\right\rangle=\left\langle\left.\mathcal{P}_{i T}\right|_{\varepsilon_{r}^{\prime}=0}\right\rangle
$$

and also that

$$
\langle\partial P \mathcal{E} / \partial t\rangle+\langle\partial K \mathcal{E} / \partial t\rangle+\left\langle\partial P \mathcal{E}_{E} / V \partial t\right\rangle=\left\langle\left.\mathcal{P}_{i T}\right|_{\varepsilon_{r}^{\prime \prime}=0}\right\rangle .
$$

The input energy per cycle is

$$
\bar{W}_{i}=\pi \varepsilon_{0} E_{0}^{2} \varepsilon_{r}^{\prime \prime}
$$

The energy dissipated per cycle is

$$
\bar{W}_{d}=\pi \varepsilon_{0} E_{0}^{2} \gamma \frac{\omega}{\omega_{p}^{2}}\left(\left(\varepsilon_{r}^{\prime}-1\right)^{2}+\varepsilon_{r}^{\prime \prime 2}\right)=\pi \varepsilon_{0} E_{0}^{2} \varepsilon_{r}^{\prime \prime}=\bar{W}_{i} .
$$

The above results are predicated on the form of the dielectric response chosen, given in Eq. (16).

\subsection{General Form}

More generally, taking into account the different orbitals and resonant frequencies of the electrons, the dielectric permittivity is of the form $[29,30]$

$$
\varepsilon_{r}=1+\sum_{j} \frac{\omega_{p j}^{2}}{\omega_{0 j}^{2}-\omega^{2}-i \gamma_{j} \omega}=1+\sum_{j} \chi_{j} .
$$

In this case, we sum over the contributions of electrons in these different orbitals, and obtain for the average stored energy density

$$
\bar{W}_{s}=\frac{1}{4} \varepsilon_{0} E_{0}^{2}\left(1+\sum_{j}\left|\frac{\partial \omega \chi_{j}}{\partial \omega}\right|\right),
$$

and for the average energy dissipated per cycle

$$
\bar{W}_{r}=\pi \varepsilon_{0} E_{0}^{2} \sum_{j} \chi_{j}^{\prime \prime}
$$

where $\chi_{j}^{\prime \prime}$ is the imaginary part of $\chi_{j}$. Again, this is equal to the input energy per cycle.

\footnotetext{
${ }^{2}$ Note that if Eq. (16) is inserted into Eq. (8), the energy density can be negative; for example, near resonance when $0<\gamma<\omega_{p}$.
} 


\section{Conclusions}

We have shown that, as suggested by Brillouin [16], the electrical energy stored in lossy dispersive materials can be regarded to consist of the energy density of the Maxwell field in vacuum, plus the sum of the kinetic and potential energies of the electrons in the material. Although we have only considered simple harmonically bound electrons, we expect our results to be applicable to other systems, such a assemblies of nanoparticles, whose electric susceptibility has the form in Eq. (32). Our results are in agreement with other microscopic approaches in the literature $[18,19,20,8,21,24,7,25]$, but are at variance with results from macroscopic approaches [22, 9, 27] including Eq. (8), which admits negative energy densities.

Our main point is that the material contribution to the electrical energy density can, in general, be viewed simply as the sum of the kinetic and potential energies of the charges in the material due to the applied electric field. We have derived simple expressions for both the stored and dissipated energies. Our results indicate that, in general, the stored electrical energy in dissipative dispersive materials cannot be written in terms of the dielectric permittivity and the field alone. In the lossless case, our expression for the stored energy reduces to the standard [15] result.

\section{Acknowledgements}

We acknowledge support by the NSF under EFRI-1332271, IIP-1114332 and DMS-1212046.

\section{References}

[1] I. Hadjipaschalis, A. Poullikkas, and V. Efthimiou. Overview of current and future energy storage technologies for electric power applications. Renewable and Sustainable Energy Reviews, 13(6-7):1513$1522,2009$.

[2] Y Gogotsi. Materials science: Energy storage wrapped up. Nature, 509:568-570, 2014.

[3] C. Yang, Y. Lin, and Nan C. Modified carbon nanotube composites with high dielectric constant, low dielectric loss and large energy density. Carbon, 47(4):1096-1101, 2009.

[4] W. Li, L. Jiang, X. Zhang, Y. Shen, and C. Nan. High-energy-density dielectric films based on polyvinylidene fluoride and aromatic polythiourea for capacitors. J. Mater. Chem. A, 2:15803-15807, 2014.

[5] S. Liu, S. Xue, W. Zhang, and J. Zhai. Enhanced dielectric and energy storage density induced by surface-modified batio3 nanofibers in poly(vinylidene fluoride) nanocomposites. Ceramics International, 40(10A):15633-15640, 2014.

[6] R.W. Ziolkowski. Superluminal transmission of information through an electromagnetic metamaterial. Phys. Rev. E, 63(4):046604, 2001.

[7] S.A. Tretyakov. Electromagnetic field energy density in artificial microwave materials with strong dispersion and loss. Phys. Lett. A, 343(1-3):231-237, 2005.

[8] A.D. Boardman and K. Marinov. Electromagnetic energy in a dispersive metamaterial. Phys. Rev. B, $73(16): 165110,2006$.

[9] K.J. Webb and Shivanand. Electromagnetic field energy in dispersive materials. Journal of the Optical Society of America B-Optical Physics, 27(6):1215-1220, 2010.

[10] R.P. Feynman, R.B. Leighton, and M.L. Sands. The Feynman lectures on physics, vol II, 27-4. AddisonWesley, Redwood City, Calif., 1989.

[11] D.J. Griffiths. Introduction to Electrodynamics, chapter 2. Prentice-Hall, New Jersey, 1st edition, 1989.

[12] M. Born and E. Wolf. Principles of optics. Cambridge University Press, Cambridge, 6th (corr.) edition, 1997. 
[13] R.D. Guenther. Modern optics. Wiley, New York, 1990.

[14] C.F. Bohren and D.R. Huffman. Absorption and scattering of light by small particles. Wiley, New York, 1983.

[15] L.D. Landau, E.M. Lifshits, and L.P. Pitaevskii. Electrodynamics of continuous media, chapter 9. Pergamon, New York, 2nd edition, 1984.

[16] L. Brillouin. Sur la propagation de la lumiere dans un milieu dispersif. C. R. Seances Acad. Sci., 23(173):1167-1170, 1921.

[17] L. Brillouin. Wave propagation and group velocity. Academic Press, New York, 1960.

[18] R. Loudon. Propagation of electromagnetic energy through an absorbing dielectric. Journal of Physics A: General Physics, 3(3):233-245, 1970.

[19] R. Ruppin. Electromagnetic energy density in a dispersive and absorptive material. Phys. Lett. A, 299(2-3):309-312, 2002.

[20] T.J. Cui and J.A. Kong. Time-domain electromagnetic energy in a frequency-dispersive left-handed medium. Phys. Rev. B, 70(20):205106, 2004.

[21] W. Shin, A. Raman, and S. Fan. Instantaneous electric energy and electric power dissipation in dispersive media. J. Opt. Soc. Am. B, 29:1048-1054, 2012.

[22] J.D. Jackson. Classical electrodynamics, chapter 6. Wiley, New York, 3rd edition, 1999.

[23] J.A. Stratton. Electromagnetic Theory, chapter 2. McGraw- Hill Book Company, New York and London, 1941.

[24] P.C.W. Fung and K. Young. Electric energy density in a dissipative medium by circuit analog. Am. J. Phys., 46(1):57-59, 1978.

[25] O.B. Vorobyev. Energy density of macroscopic electric and magnetic fields in dispersive medium with losses. Progress In Electromagnetics Research B, 40:343-360, 2012.

[26] J. Askne and B. Lind. Energy of electromagnetic waves in the presence of absorption and dispersion. Phys. Rev. A, 2(6):2335-2340, 1970.

[27] F.D. Nunes, T.C. Vasconcelos, M. Bezerra, and J. Weiner. Electromagnetic energy density in dispersive and dissipative media. J. Opt. Soc. Am. B, 28(6):1544-1552, 2011.

[28] P. Grujic and N. Simonovic. Insights from the classical atom. Physics Today, 65(5):40, 2012.

[29] G.R. Fowles. Introduction to modern optics. Holt, New York, 1968.

[30] M.V. Klein and T.E. Furtak. Optics. Wiley, New York, 2nd edition, 1986. 